\title{
Untersuchung der dynamischen Strukturverformung von Werkzeugmaschinen mit Tracking-Interferometern
}

\author{
Prof. Dr.-Ing. C. Brecher ${ }^{1}$, Dipl.-Ing. Dipl.-Kfm. A. Guralnik ${ }^{1}$, Dipl.-Phys. M. Wissmann ${ }^{2}$, Dipl.-Ing. (FH) D. \\ Berk $^{3}$, Dr.-Ing. H. Schwenke ${ }^{2}$, Dipl.-Ing (FH) H. Paluszek ${ }^{3}$ \\ ${ }^{1}$ Lehrstuhl für Werkzeugmaschinen, Werkzeugmaschinenlabor WZL der RWTH Aachen \\ Steinbachstraße 19, 52074 Aachen \\ ${ }^{2}$ Etalon AG, Bundesallee 100, Braunschweig 38116 \\ ${ }^{3}$ sigma3D, Max-Hufschmidt-Straße 4a, Mainz 55130
}

Tel.-Nr.: +49 (0) 241-80 27440, E-Mail: a.guralnik@wzl.rwth-aachen.de

\section{Einleitung}

Bei der spanenden Bearbeitung von Werkstücken auf Werkzeugmaschinen treten Schwingungen der Maschinenstruktur auf, die die nutzbare Maschinenleistung deutlich reduzieren können. Bei der Entwicklung von Werkzeugmaschinen und teilweise für die Prozessoptimierung ist es daher erforderlich, mit Hilfe experimenteller Untersuchungen der dynamischen Strukturverformung, Schwachstellen der Maschinenstruktur aufzudecken. Diese Untersuchung, die sogenannte Modalanalyse, umfasst die Messung der Schwingungen an bis zu dreihundert Punkten der Maschine in drei Dimensionen und die mathematische Ableitung der Eigenformen. Zwar steigt mit dem rasanten Wachstum der Rechenleistung auch die Bedeutung der Simulation, doch scheint diese die experimentelle Modalanalyse nur zusätzlich zu fördern [1].

Hauptanwendungsgebiet der Modalanalyse sind die Charakterisierung von Materialeigenschaften, Abgleich theoretischer Modelle und Simulationsergebnisse sowie, insbesondere vor dem Hintergrund der Anwendungsbeispiele in diesem Artikel, die Identifikation von mechanischen Strukturschwachpunkten im Betrieb [1]. Anhand der Nachgiebigkeitsfrequenzgänge kann die Neigung der Maschine zu Instabilitäten beurteilt sowie kritische Frequenzen identifiziert werden $[2,3]$. Jedoch erlaubt nur die Modalanalyse die Identifikation der Schwachpunkte der Maschinenstruktur anhand derer, Verbesserungspotentiale abgeleitet werden können [4]. Der starke Bezug zu Werkzeugmaschinen liegt im Schwerpunkt unserer Forschungs-, Entwicklungs-, und Dienstleistungsaktivitäten. Die meisten Aussagen dieses Artikels sind jedoch genauso gültig für die Modalanalyse von Fahrzeugen, Flugzeugen, Musikinstrumenten und Ähnlichem sowie teilweise für die Modalanalyse von Gebäuden, Brücken usw.

Abhängig von der Art der zu untersuchenden Struktur werden hauptsächlich Beschleunigungssensoren und Laservibrometer für Modalanalysen verwendet. Sogenannte 3D-ScanningVibrometer werden vorwiegend bei offenen Oberflächen und dünnen Strukturen in der Automobil- und Luftfahrtindustrie eingesetzt [5]. Die drei Laser-Köpfe des Gerätes werden auf das zu messende Objekt gerichtet, die Messung erfolgt berührungslos und sehr zügig über große Flächen, braucht jedoch einen großen Sichtkorridor. Wegen der Sichtlinienproblematik werden in umhausten oder teilweise verdeckten Strukturen triaxiale Beschleunigungssensoren verwendet [2, 4]. Die zügige Messung mit Vibrometern steht der Flexibilität der Beschleunigungssensoren gegenüber, außerdem weisen einige Forschungsarbeiten den Beschleunigungssensoren bessere Ergebnisse zu [6]. Schließlich sind Systeme auf Basis von Beschleunigungssensoren wesentlich kostengünstiger und in der Regel kompakter.

\section{Motivation zur Entwicklung neuer Sensoren und Messtechniken}

Die Forschungsgruppe Maschinenuntersuchung und -beurteilung am Lehrstuhl für Werkzeugmaschinen am des WZL führt seit den 80er Jahren Modalanalysen nicht nur in der Forschung sondern insbesondere als industrielle Dienstleistung durch. In den vergangenen 20 Jahren wurden mehr als 300 Werkzeugmaschinen unterschiedlicher Art und Größe untersucht. Modalanalysen an Werkzeugmaschinen werden heute mit Hilfe von triaxialen Beschleunigungssensoren 
durchgeführt. Nach unserer Erfahrung besteht die Modalanalyse von Werkzeugmaschinen onsite in der Regel aus drei Schritten: Modellierung der Maschinengeometrie, Schwingungsmessung und Datenprüfung. Modalanalysen typischer Dreh-Fräs-Bearbeitungszentren werden aus Messungen an 200 bis 300 Punkten erstellt. Bild 1 zeigt den relativen Zeitaufwand der einzelnen Schritte.

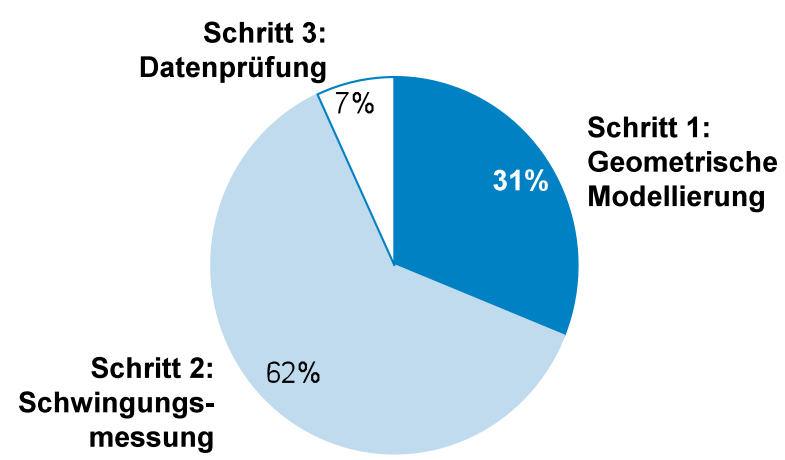

Bild 1: Zeitverteilung für die Modalanalyse eines typischen Bearbeitungszentrums

Bei der Modellierung wird die zu messende Struktur durch ein vereinfachtes geometrisches Modell aus diskreten Messpunkten angenähert. Die absolute Lage der Punkte wird mit Hilfe eines Maßbandes vermessen, in einen Computer eingegeben und mit Verbindungslinien zu einem Netz-Modell vervollständigt. Die Modellierung kann meist aus Geheimhaltungsgründen, schwieriger Einschätzung der physischen Zugänglichkeit der Messpunkten oder Dringlichkeit der Aufträge nicht anhand von CAD-Daten oder Ähnlichem vorab erfolgen. Bei der Schwingungsmessung wird ein Beschleunigungssensor an den einzelnen ausgewählten Punkten der Maschinenstruktur nach einander befestigt, zu den Maschinenkoordinaten ausgerichtet, die aktuelle Sensororientierung im globalen Koordinatensystem wird in einem ModalanalyseProgramm angegeben und schließlich werden die Nachgiebigkeitsfrequenzgänge vermessen. Im letzten Schritt der Modalanalyse, der Datenprüfung, werden die Daten vor Ort beurteilt, eventuelle Inkonsistenzen aufgedeckt und Fehlmessungen wiederholt. Dagegen erfolgt die genaue Analyse off-site.

Den mathematischen Kern der Modalanalyse, das sogenannte curve-fitting, die Eigenfrequenz-, die Schwingungsform-Berechnung und die Visualisierung beinhaltet der dritte Schritt. Er ist in den meisten kommerziellen Softwaresystemen jedoch weitgehend automatisiert und mit einem Minimum an manuellen Tätigkeiten verbunden. Die ersten beiden Schritte (Modellierung und Schwingungsmessung) sind dagegen weitgehend manuell, auch mit viel Aufwand kaum automatisierbar und sehr zeitintensiv. Gerade hier verbirgt sich nach unserer Auffassung ein großes Potenzial.

\section{Tracking-Interferometer}

Das Laser-Tracking-Interferometer (TI) wurde im amerikanischen national bureau of standards (NBS) in den 1980er Jahren als optisches Kugelkoordinatenmessgerät zur Kalibrierung von Robotern entwickelt und patentiert [7]. Wie in Bild 2 dargestellt wird bei einem TI ein Laserstrahl über zwei Drehachsen geführt und auf einen Reflektor gelenkt, dem das Gerät folgen kann (Tracking). Mit Hilfe der gemessenen Distanz zwischen dem Gerät und dem Reflektor und der beiden Drehwinkel $(\varphi, \theta)$ kann die Position des Reflektors im Raum eindeutig bestimmt werden. Wenn der Reflektor aus der Strahlachse wandert, entsteht ein Lateralversatz zwischen dem ausgesandten und dem reflektierten Laserstrahl. Dieser Versatz wird detektiert und als Eingangsgröße für die Folgefunktion (Tracking) genutzt. Heute werden die Geräte unter dem Namen Lasertracker von den Firmen Faro, Leica und API bzw. seit einigen Jahren als LaserTracer von der Firma etalon hergestellt und vertrieben. Ursprünglich wurde die Distanz stets interferometrisch ausgehend von einer bekannten Position ohne Strahlunterbrechung bis zur Messposition vermessen. Heutige Lasertracker verfügen meist über eine Time-of-Flight-basierte Absolut- 
distanzmesseinheit (ADM) die ohne die Referenzposition auskommt. Der Nutzungsschwerpunkt der Lasertracker liegt in der geometrischen Messung.

Die in Bild 2 dargestellte Variante des TI ist der sogenannte LaserTRACER. Das Gerät ist aus einander Zusammenarbeit der britischen national physical laboratory (NPL) mit der deutschen Physikalisch-Technischen-Bundesanstalt (PTB) hervorgegangen und wird von der Firma etalon vertrieben. Die Genauigkeit der relativen Längenmessung des LaserTRACERs ist durch die besondere Strahlführung so hoch, dass mit Hilfe der Multilaterationstechnik sogar Koordinatenmessgeräte kalibriert werden können. Die Strahlführung und der Einfluss der Achsfehler auf die Messgenauigkeit ist in [8] beschrieben, in [9, 10] wird der Eignungsnachweis des LaserTRACERs für die Kalibrierung von Koordinatenmessgeräten und Werkzeugmaschinen erbracht.

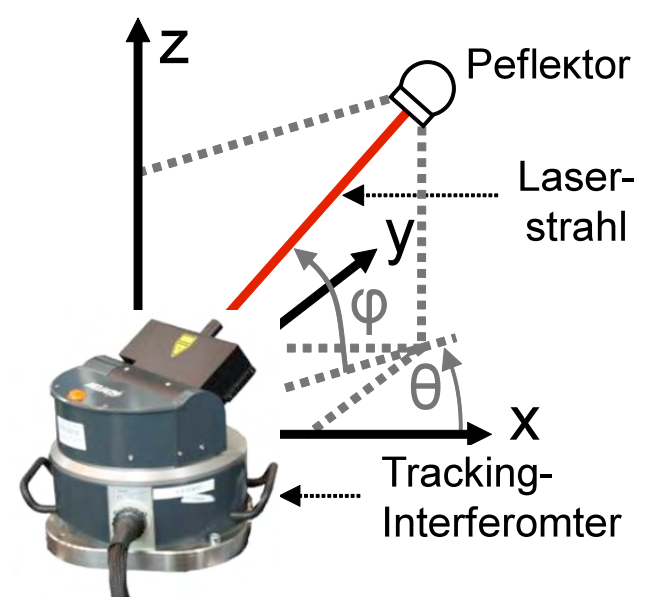

Bild 2: Tracking-Interferometer mit Koordinatensystem und Reflektor

Werkzeugmaschinen sind sehr steife Strukturen und können auch mit den stärksten Aktoren nur um einige Mikrometer verformt werden. Typischerweise müssen im Bereich von bis zu $1000 \mathrm{~Hz}$ Verformungen ab etwa 300 nm detektiert werden. Die Messunsicherheit der 3DMessungen mit Tracking-Interferometern ist größer al $10 \mu \mathrm{m}$ und steigt mit dem Abstand zum Reflektor. Der limitierende Faktor ist dabei die Winkelmessung. Zusätzlich ist weder die Absolutdistanzmesseinheit (ADM) noch die Tracking-Einheit zur Messung solch schneller Bewegungen geeignet.

Der Ansatz zur Durchführung von Modalanalysen mit dem Tracking-Interferometer ist zuerst in [11] beschrieben worden und liegt in der Trennung der Absolutmessung anhand der Absolutdistanz und zweier Drehwinkel von der Dynamikmessung. Die Dynamikmessung erfolgt relativ mit Hilfe des Interferometers und der positionssensitiven Diode PSD. Beide Einheiten sind sehr hochauflösend im Submikrometerbereich und sehr schnell.

\section{Hochdynamische 3D-Messung mit einem Laserstrahl}

Mit Hilfe des Tracking-Interferometers und eines Reflektors können hochdynamische Messungen mit einem Laserstrahl in allen drei Dimensionen ausgeführt werden. Grundsätzlich bleibt die Sichtlinienproblematik des 3D-Vibrometers hierbei erhalten, jedoch begnügt sich das einstrahlige TI mit wesentlich schmaleren Sichtkorridoren. Auf der anderen Seite misst das Gerät durch die Notwendigkeit des Reflektors nicht völlig berührungslos.

Bild 3 zeigt ein detailliertes Schema des Strahlengangs innerhalb eines sogenannten optischen Retroreflektors. Der Retroreflektor wirft den unter einem (in bestimmten Grenzen) beliebigen Winkel einfallenden Laserstrahl parallel zurück. Trifft der Laserstrahl mit einem Versatz zur Mitte des Reflektors ein, wird dieser wie gezeigt mit dem doppelten Lateralversatz jedoch weiterhin parallel zurückgeworfen. Die Bewegung in Strahlrichtung kann somit mit dem Interferometer und die quer zur Strahlrichtung mit der PSD erfasst werden. Da die Reflexion stets parallel er- 
folgt, sinkt die Auflösung der Lateralmessung nicht mit dem Abstand zwischen TI und Reflektor, wie es bei der Winkelmessung der Fall ist.

Anhand des Schemas in Bild 3 lässt sich zeigen, dass die in-Strahlbewegung, die Lateralbewegung und die Reflektordrehung sich nicht gegenseitig beeinflussen. So wird von einem vernachlässigbar geringem Übersprechverhalten ausgegangen.

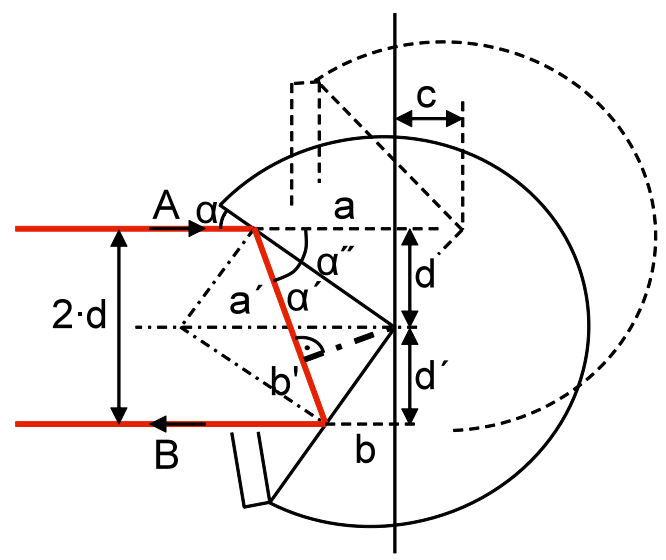

Bild 3: Strahlengang innerhalb eines optischen Retro-Reflektors

Der eigentlich dreidimensionale Retroreflektor (Trippelspiegel) kann ohne Einschränkung der Allgemeingültigkeit auch in zwei Dimensionen (Doppelspiegel) analysiert werden (vgl. Bild 3). Sei der Grundzustand des Reflektors so gewählt, dass der Laserstrahl auf der Winkelhalbierenden zwischen den beiden Spiegeln liegt. Eine Verdrehung des Reflektors im Grundzustand verursacht offensichtlich weder eine Strahllängenänderung noch einen Lateralversatz. Die Verschiebung des Reflektors in Strahlrichtung um $\mathrm{c}$ in der Grundstellung verursacht ebenfalls keinen Strahlenversatz. Wenn die Strahllänge bei Lateralversatz konstant bleibt, ist das System frei von Einflüssen des Übersprechens, weil sich jede Zustandsänderung (also z.B. eine Verdrehung und in Strahlverschiebung des Reflektors aus einem anderen als dem Grundzustand) als Lateralverschiebung in die Strahlachse, Drehen und In-strahl-Bewegung und anschließende laterale Rückverschiebung erklären lassen.

Eine Lateralverschiebung des Reflektors um d führt zu einem Strahlenversatz von $2 \cdot d$. Dies ist in Bild 3 anhand eines verschobenen und verdrehten Reflektors dargestellt und ist geometrisch damit erklärbar, dass die beiden Lote in den Reflektionspunkten zusammen mit den Spiegelflächen ein Rechteck bilden, durch dessen Diagonale der Laserstrahl zwischen den Spiegeln verläuft. Die andere Diagonale und somit der Mittelpunkt des Rechtecks liegt immer auf der Winkelhalbierenden zwischen den beiden Spiegeln. Die Konstanz der Strahllänge bei Lateralversatz wird wie folgt erklärt: die Strahllänge beträgt $2 \cdot(A+a)=2 \cdot(B+b)$ wenn der Strahl auf das Reflektorzentrum gerichtet ist. Zu beweisen ist also $a^{\prime}=a$ und b' $=b$ und der Beweis wird mit Hilfe von ähnlichen Dreiecken erbracht. Mit den Grundgleichungen der Reflektion folgt $\alpha=\alpha^{\prime}$ und weiterhin $\alpha=\alpha^{\prime \prime}$. Da $\alpha^{\prime}=\alpha^{\prime \prime}$ sind die beiden rechtwinklige Dreiecke mit der gemeinsamen Hypotenuse an der oberen Spiegelfläche genau spiegelsymmetrisch, was unmittelbar zu a = a' führt. Aus dem gleichen Grund gilt auch $b=$ b'. Diese beiden Zusammenhänge gelten auch bei gedrehter Reflektorstellung.

\section{Vorgehensweise zur Messung teilumhauster Strukturen mit einem TI}

Bild 4 zeigt den Entwurf einer Messstrategie zur Durchführung von Modalanalysen an teilumhausten Strukturen mit Tracking-Interferometern. Generell handelt es sich um die parallele Durchführung der Schritte „geometrische Modellierung“ und „Schwingungsmessung“. Es werden zwei Sätze von Messreflektoren und ein Satz Referenzreflektoren benutzt.

Der erste Satz der Messreflektoren wird so an verschiedenen Punkten der Maschinenstruktur verteilt, dass sie für das TI in seiner ersten Messposition sichtbar sind. Es handelt sich um einen der zeitlich aufwändigsten Schritte. Die Positionen der Reflektoren werden vom Benutzern 
nen der zeitlich aufwändigsten Schritte. Die Positionen der Reflektoren werden vom Benutzern nacheinander mit dem TI angepeilt (Teachen). So liegen die absoluten Koordinaten der Punkte direkt für das Geometriemodell vor und müssen vom Bediener lediglich noch zu einem Netzmodell verbunden werden. In dieser Zeit läuft bereits die vollautomatisierte Messung, bei der das TI die eben geteachten Punkte nacheinander anpeilt und die Schwingungsmessung durchführt. Der Nutzer bringt in den Satz der Referenzreflektoren und einen zweiten Satz der Messreflektoren an. Durch das Einmessen der Referenzreflektoren aus der aktuellen und der nächsten TIMessposition wird die Pose des TI, das heißt die Position und die Orientierung, bestimmt, so dass Messungen aus unterschiedlichen Messpositionen in ein gemeinsames Koordinatensystem transformiert werden. Sinnvollerweise werden die Referenzkorridore an Punkten befestigt, die aus sehr vielen Messpositionen sichtbar sind, so wird zusätzlich Zeit eingespart und die Fehlerfortpflanzung minimiert.

Weiterhin bilden die manuellen Tätigkeiten den zeitlich kritischen Pfad der Messung. Die wichtigsten Zeit-Einsparpotentiale liegen in der verkürzten und weitgehend integrierten Geometrieerstellung, in der Parallelisierung der vollständig automatisierten Messung und im Wegfall der Angabe der Sensororientierung vor jeder Messung.

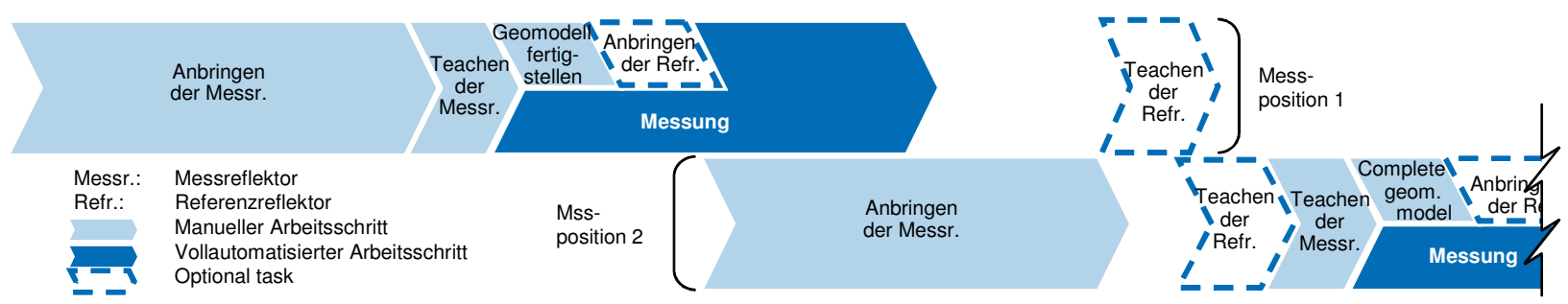

Bild 4: Vorgehensweise bei der Durchführung von Modalanalysen mit einem Tracking-Interferometer

\section{Eindimensionale Vergleichsmessungen, Benchmark}

Im folgenden Beispielen wird der LaserTRACER der Fa. etalon verwendet. Das Gerät verfügt über einen frequenzstabilisierten He-Ne-Laser-Interferometer und eine positionssensitive Diode. Die in-Strahl-Bewegung wird direkt über das analoge Sinus-Kosinus-Signal des Interferometer aufgenommen. Mit der Zirkularinterpolation der Signale kann leicht eine Auflösung von einem Nanometer erreicht werden. Die maximale messbare Geschwindigkeit wird durch die verwendete Hardware auf etwa $15 \mathrm{~mm} / \mathrm{s}$ beschränkt, welche bei Messungen an Werkzeugmaschinen meistens bei Weitem nicht erreicht wird. Die verwendete positionssensitive Diode in Kombination mit der Optik und der verwendeten Elektronik erreicht eine Signalverstärkung von $2.19 \mathrm{~mm} / \mathrm{V}$. [11] In Bild 5 sind der Messaufbau und die mit verschiedenen Sensoren gemessenen Nachgiebigkeitsfrequenzgänge einer Fahrständermaschine dargestellt. Es handelt sich - der Legendenreihenfolge nach - um Messungen mit einem induktiven Wegsensor, der TI-PSD, einem LaserDopplervibrometer, einem Beschleunigungssensor und dem TI-interferometer. Der direkte Nachgiebigkeitsfrequenzgang ist in x-Richtung aufgenommen worden. Hierzug wurde das $\mathrm{TI}$ an zwei verschiedenen Stellen außerhalb der Maschine positioniert, wie das Prinzipbild links unten andeutet. Das Laser-Dopplervibrometer wurde außerhalb der Maschine befestigt. Die Wegsensoren wurden relativ zwischen Tisch und Werkzeug positioniert und deren Messung mit einer absoluten Messung der Tischbewegung verrechnet. 

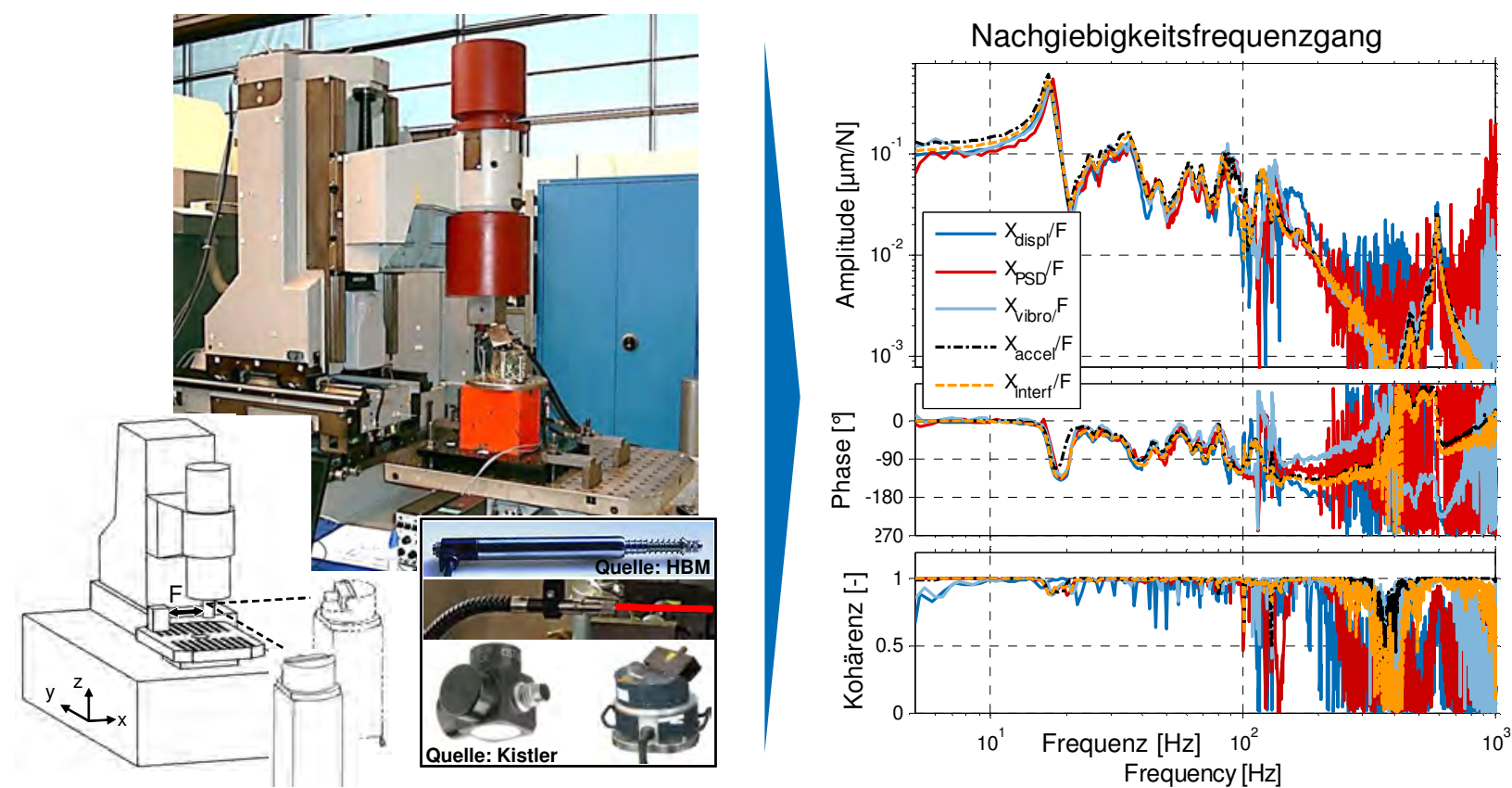

Bild 5: Messaufbau und Messergebnisse der Nachgiebigkeitsfrequenzmessung einer Fahrständermaschine

In Bild 6 sind die Messungen aus Bild 5 mit einem vertikalen Versatz der Kurven dargestellt. Anhand der Form des Amplitudenganges und insbesondere am Kohärenzverlauf, kann die Güte der Messungen beurteilt werden. Generell lässt sich sagen, dass zwischen $10 \mathrm{~Hz}$ und $100 \mathrm{~Hz}$ alle Sensoren sehr gute Messungen zulassen. Im Bereich um $400 \mathrm{~Hz}$ brechen die Kohärenzen aller Sensoren ein. Während die Kohärenz beim Interferometer und bei Beschleunigungssensoren auf einem Band von nur ca. $100 \mathrm{~Hz}$ unmittelbar um die Antiresonanz herum einbricht, ist sie für Wegsensoren und die PSD auf einem Band von ca. $250 \mathrm{~Hz}$ bis zur Resonanz bei ca. $600 \mathrm{~Hz}$ als schlecht zu bezeichnen. Nach der Resonanzstelle bricht die Kohärenz wiederum ein. Bild 6 rechts trägt das Signal-Rausch-Verhältnis über der Frequenz auf. Das Rauschniveau wurde während einer Ruhemessung abgelesen und durch die Quadratwurzel aus dem Autoleistungsspektrum der Messung geteilt. Werte über eins bedeuten ein höheres Rauschniveau als das Nutzsignal.
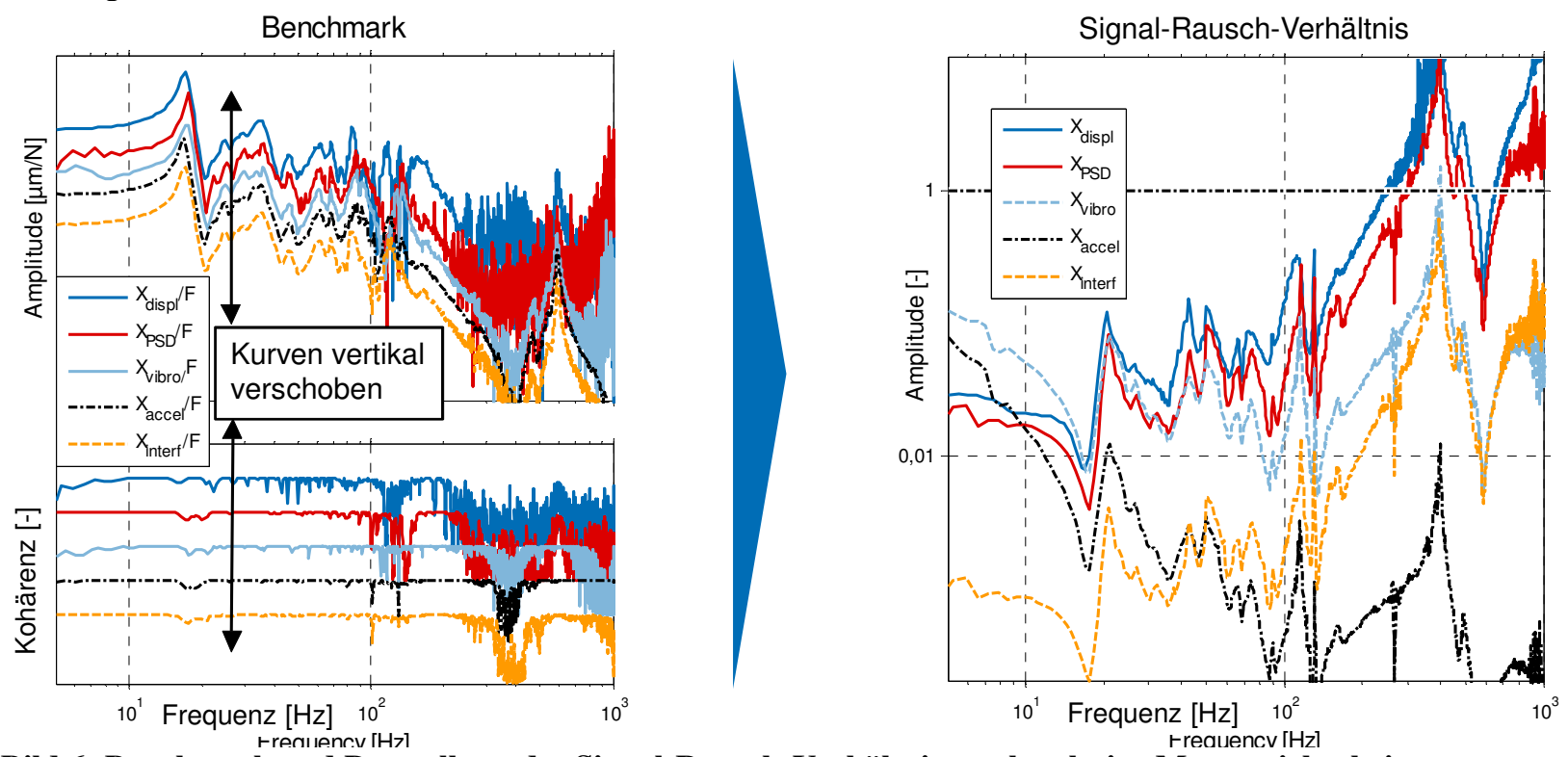

Bild 6: Benchmark und Darstellung des Signal-Rausch-Verhältnisses als relative Messunsicherheit

Im Bereich niedriger Frequenzen zeigen Interferometermessungen deutlich die geringste Messunsicherheit auf. Mit steigender Frequenz zeigt die Messunsicherheit der Beschleunigungssen- 
soren die geringsten Werte auf, weil die Beschleunigung bei gleicher Wegamplitude quadratisch mit der Frequenz steigt. Im Falle des Laservibrometers, welches Geschwindigkeiten misst, liegt eine lineare Abhängigkeit von der Frequenz vor. Unterhalb von $10 \mathrm{~Hz}$ ist die relative Messunsicherheit bereits höher als bei anderen Verfahren. Entsprechend der Kohärenz steigen die relativen Messunsicherheiten beider Signale im Bereich zwischen $250 \mathrm{~Hz}$ und ca. $600 \mathrm{~Hz}$ sowie hinter der Resonanzstelle über den Wert eins. Unmittelbar an der Resonanzstelle selbst liegen die Kurven der Messunsicherheit für alle Sensoren unter eins und die Kohärenz nahe bei eins. Das heißt, dass die Resonanzhöhe von allen Sensoren zuverlässig bestimmt wird.

Das Rauschniveau der PSD beträgt umgerechnet ca. $4 \mu \mathrm{m}$, das Rauschen ist jedoch sehr stark mit einem der beiden Interferometersignale korreliert, also nicht zufällig, was auf unerwünschte Mehrfachreflektionen im Strahlengang zurückzuführen ist. Auf der einen Seite können verschiedene Auswertetechniken zur Unterdrückung dieser Fehleranteile angewendet werden [13] auf der anderen Seite konnte der Rauschanteil durch das Abschirmen eines Teils des Laserstrahls sehr einfach auf $1 \mu \mathrm{m}$ reduziert werden, was jedoch zeitlich getrennte Messungen mit PSD und Interferometer erfordern würde. Die Verbesserung der optischen Komponenten ist Gegenstand künftiger Forschungsanstrengungen.

\section{D-Messung und Koordinatentransformation}

Für räumliche Messungen mit dem Tracking-Interferometer müssen drei verschiedene Koordinatensysteme definiert werden. Das globale Koordinatensystem in Bild 7 bezieht sich typischerweise auf das Maschinenkoordinatensystem und ist für alle durchzuführenden Messungen gleich. Das Basiskoordinatensystem bezieht sich auf die Basis des Tracking-Interferometers und ist für jede Position des TI separat definiert. Das TI-Koordinatensystem wird durch die Ausrichtung des Laserstrahls und der PSD definiert und ist für jeden Reflektor und, falls ein Reflektor aus verschiedenen Positionen vermessen wird, zusätzlich für jede Position unterschiedlich.

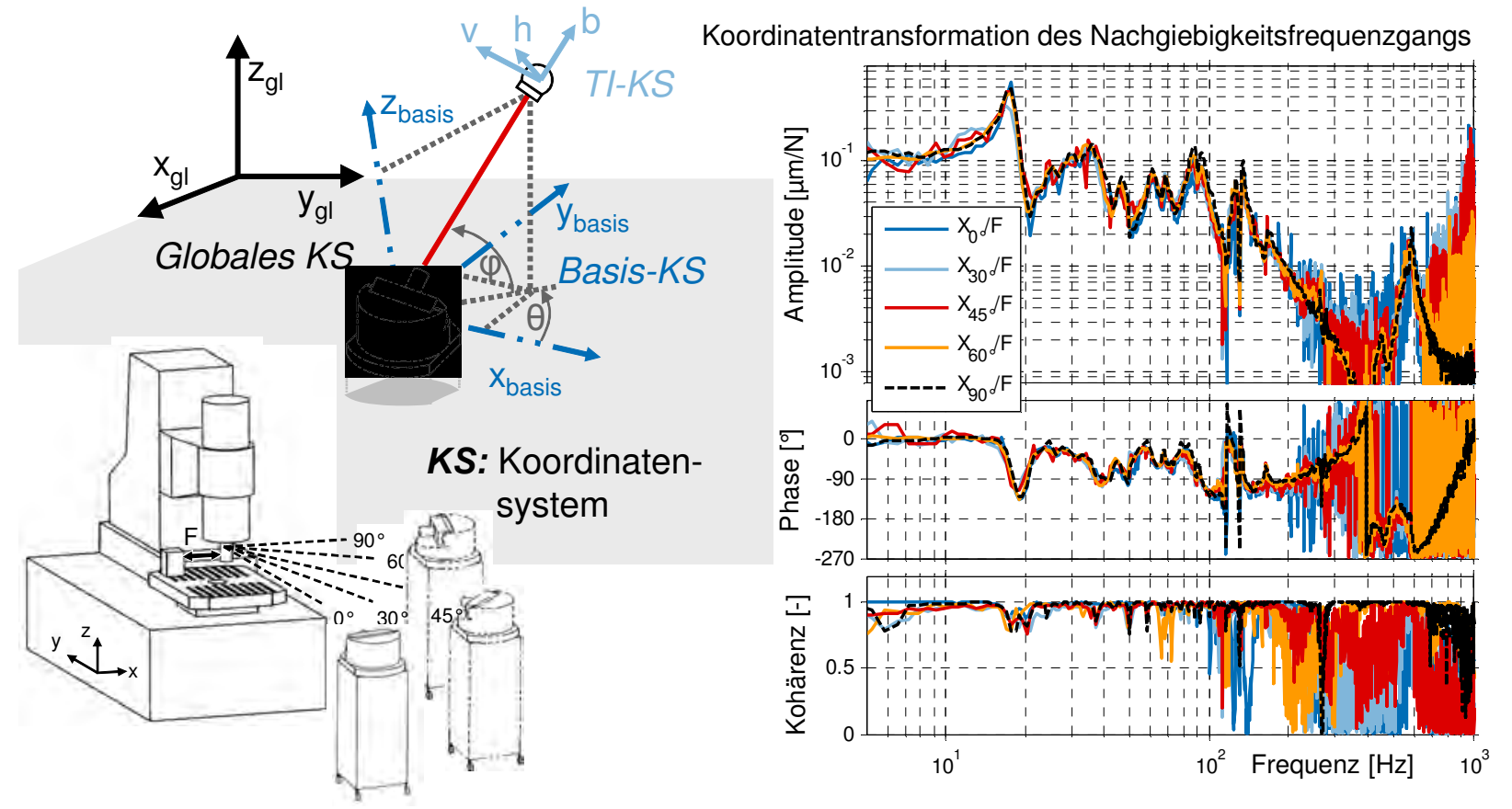

Bild 7: Koordinatentransformation der Dynamikmessungen aus unterschiedlichen Richtungen

Anhand der Messungen in Bild 7 wird die Koordinatentransformation des Messsignals erläutert. Links unten ist der Aufbau für die Messung des direkten Nachgiebigkeitsfrequenzganges in $\mathrm{x} \square$ Richtung der Maschine dargestellt. Der Reflektor wird hierbei aus unterschiedlichen Richtungen angepeilt. Das Stativ des TI wird immer gleich ausgerichtet, so dass die Winkellage zwischen Maschine und TI direkt von den Winkelencodern ausgelesen werden kann. Im rechten 
Teil sind die Messergebnisse dargestellt. Bei der Messung quer zur Strahlrichtung $\left(0^{\circ}\right)$ wird nur die PSD-Messung ausgewertet, bei $90^{\circ}$ nur die interferometrische Messung bei anderen Winkelstellungen handelt es sich um eine Kombination aus beiden. Die Berechnungsvorschrift wird in [11] erläutert, außerdem wird gezeigt, dass die Koordinatentransformation sowohl im Zeit- als auch im Frequenzbereich durchgeführt werden kann. Dabei ist die Koordinatentransformation im Frequenzbereich bei typischerweise fünf bis zehn Mittelungen um den Faktor 2,5 bis 20 weniger rechenintensiv.

Typischerweise können nicht alle Messpunkte von einer einzigen Position des $\mathrm{TI}$ aus angepeilt werden. Um alle Messungen in ein gemeinsames Koordinatensystem überführen zu können, muss die relative Pose des $\mathrm{TI}$ in den verschiedenen Messpositionen bekannt sein. Dieses Problem lässt sich auf verschiedene insbesondere aus der Geodäsie bekannte Fragestellungen zurückführen. Mögliche Lösungen und das verwendete Verfahren wurden in [12] dargestellt.

\section{Alternative Absolutdistanzmessung}

Bei der Absolutdistanzmesseinheit und dem Interferometer der Lasertracker handelt es sich um separate Systeme, mit verschiedenen Laserquellen, Sensoren und Auswerteeinheiten [4]. Die Absolutdistanzmesseinheit wird für dynamische Messungen nicht verwendet und der LaserTRACER von etalon verfügt über keine solch Silbentrennungs-Einheit. Für Modalanalysen mit TI wurde deswegen untersucht, ob auf eine Absolutdistanzmessung verzichtet werden kann. Fraglich ist in diesem Zusammenhang, ob die Messunsicherheit eines alternativen Verfahrens hinreichend klein ist.

Die Absolutdistanzmessung wird lediglich für den Aufbau eines Geometriemodells benutzt, das bei Messungen mit Beschleunigungssensoren mit einem Maßband eingemessen wird. Für Standardbearbeitungszentren mit einer Grundfläche von ca. $4 \times 3 \mathrm{~m}^{2}$ scheint eine Messunsicherheit von $\pm 30 \mathrm{~mm}$ als völlig ausreichend.

Zur Vermeidung zusätzlicher Kosten und potentiellen Fehlerquellen von ADM wird eine Messung der Absolutdistanz über die Strahlauslenkung vorgeschlagen. Wie in Bild 8 links dargestellt, führt eine kleine Verdrehung des Interferometer-Kopfes zu einem Lateralversatz des zurückgeworfenen Laserstrahls von $2 \mathrm{~d} \cdot \sin (\Delta \varphi)$, der mit der PSD gemessen werden kann. Dabei ist $d$ der Abstand des Reflektors vom Interferometer und $\Delta \varphi$ der Auslenkwinkel des Laserstrahls. Die maximale Auslenkung des Laserstrahls ist wegen der PSD-Größe auf ca. $\pm 1,5 \mathrm{~mm}$ beschränkt, während die Distanz zum Reflektor typischerweise mindestens ein Meter beträgt. Aus diesem Grund kann von einem linearen Zusammenhang zwischen Auslenkwinkel und PSD-Auslenkung ausgegangen werden.

Der Auslenkwinkel wurde in verschiedenen Versuchen auf zwei unterschiedliche Weisen gemessen. Bei der beschleunigungsreferenzierte Messung (Bild 8, oben) wurde die TrackingFunktion des TI mit leicht instabilen Parametern betrieben, so dass sich leichte Schwingungen bildeten. Die Schwingungen wurden einerseits über die PSD und andererseits über ein Beschleunigungssensor am Interferometer-Kopf erfasst. Der Beschleunigungssensor am Interferometer-Kopf wurde nicht verschoben, weswegen das Beschleunigungssignal der Winkelbeschleunigung proportional ist. Nach doppelter Integration im Frequenzbereich ist der Absolutwert der Übertragungsfunktion von Beschleunigung auf die PSD-Spannung proportional zur Absolutdistanz des TI zum Reflektor. Der Wert der Übertragungsfunktion wird an der Resonanzstelle von $3,5 \mathrm{~Hz}$ ermittelt und für die weitere Analyse verwendet.

Bei der geschwindigkeitsreferenzierten Messung (Bild 8, unten) wird der Laserstrahl durch den Reflektor durchgeschwenkt. Es wird davon ausgegangen, dass der Schwenkwinkel so groß ist, dass die Winkelgeschwindigkeit der Interferometer-Kopfes im Bereich, wo der Laserstrahl die PSD überfährt, konstant ist. In diesem Fall ist die Geschwindigkeit der Diodenspannung proportional zum Abstand des Reflektors und wird für die weitere Analyse verwendet. Die dritte Methode die winkelreferenzierte Absolutmessung würde direkt die Winkelstellung als Referenz nehmen, ist jedoch bisher noch nicht getestet worden. 

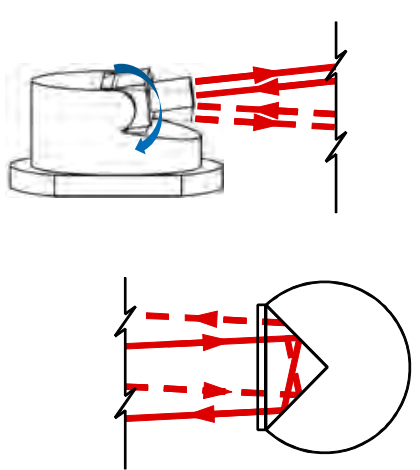

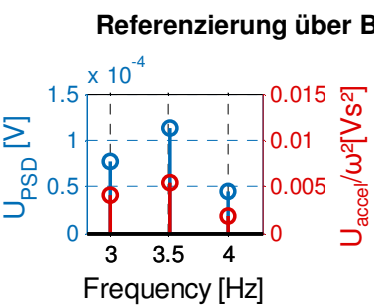

Referenzierung mit Geschwindigkeit :

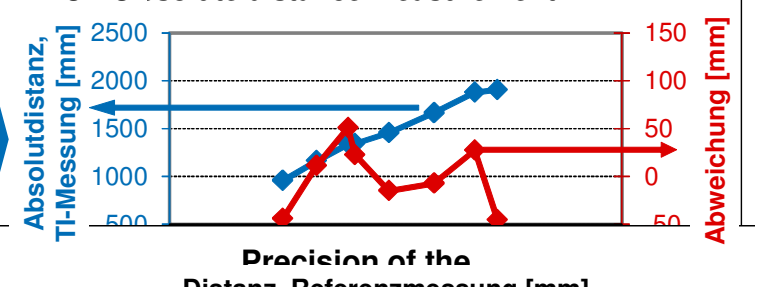

Distanz, Referenzmessung [mm]
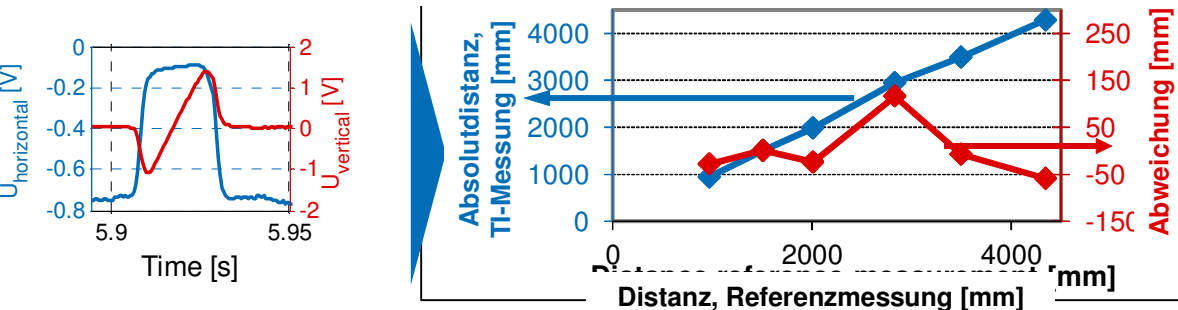

Bild 8 Absolutdistanzmessung über Beschleunigung und Geschwindigkeit

Das Verhältnis zwischen der Absolutdistanz und der beschriebenen Übertragungsfunktion bzw. der erläuterten Geschwindigkeit wird als linear modelliert und die Abweichungen vom linearen Zusammenhang als Fehler gewertet. Bild 8 rechts zeigt eine gute Linearität des Modells. Die Abweichungen bei der beschleunigungsreferenzierten Messung betragen ca. $\pm 50 \mathrm{~mm}$ bei einem getesteten Messbereich von $1-2 \mathrm{~m}$, bei der geschwindigkeitsreferenzierten Messung sind es -50 bis $150 \mathrm{~mm}$ bei einem getesteten Messbereich von 1-4,8 m. Auf dem Bereich von $1-$ $2 \mathrm{~m}$ liegt die Abweichung der geschwindigkeitsreferenzierten Messung bei unter $\pm 30 \mathrm{~mm}$. Bei den zukünftigen Versuchen, wird die Drehgeschwindigkeit der Achsen zur geschwindigkeitsreferenzierten Distanzmessung herabgesetzt. Es wird vermutet, dass hierdurch die geforderte Messunsicherheit von unter $\pm 30 \mathrm{~mm}$ bei einer Messentfernung von bis $5 \mathrm{~m}$ erreicht wird.

\section{Beispiel einer kleinen Modalanalyse mit dem Tracking-Interferometer}

Eine erste stark reduzierte Modalanalyse einer Fahrständer-Fräsmaschine ist in Bild 9 dargestellt. Die Maschinengeometrie ist mit nur 8 Punkten approximiert. Alle Punkte sind aus einer Messposition heraus vermessen worden. Für diese Modalanalyse wurde die Maschine mit Hilfe eines elektrohydraulischen Relativerreger zwischen Werkzeug und Maschinentisch mit einer dynamischen Kraft belastet. Die Geometrieerstellung wurde hierbei noch traditionell durchgeführt. Die ermittelten Eigenformen sind für die ersten beiden Eigenfrequenzen von $17 \mathrm{~Hz}$ und $34 \mathrm{~Hz}$ dargestellt. Dieser erste Test bestätigt die Funktionsfähigkeit der Schwingungsmessung und der Koordinatentransformation.

Die erste Mode ist eine Aufstellschwingung der Maschine. Die Struktur kippt um die yAchse mit den höchsten Bewegungsamplituden an den Punkten 1 und 4. Die zweite Mode ist eine Drehschwingung des Fahrständers mit einem leichten Kippen der Spindel. Die höchsten Amplituden werden am Tool-Center-Point erreicht. Hierbei handelt es sich um eine deutlich komplexe Eigenform, das heißt in diesem Fall, dass die größte Auslenkung der werkzeugseitigen Maschinenstruktur bei einer anderen Phasenlage erreicht wird, als die des Tisches. Auch dieser Effekt konnte mit dem $\mathrm{TI}$ genau herausgestellt werden. 

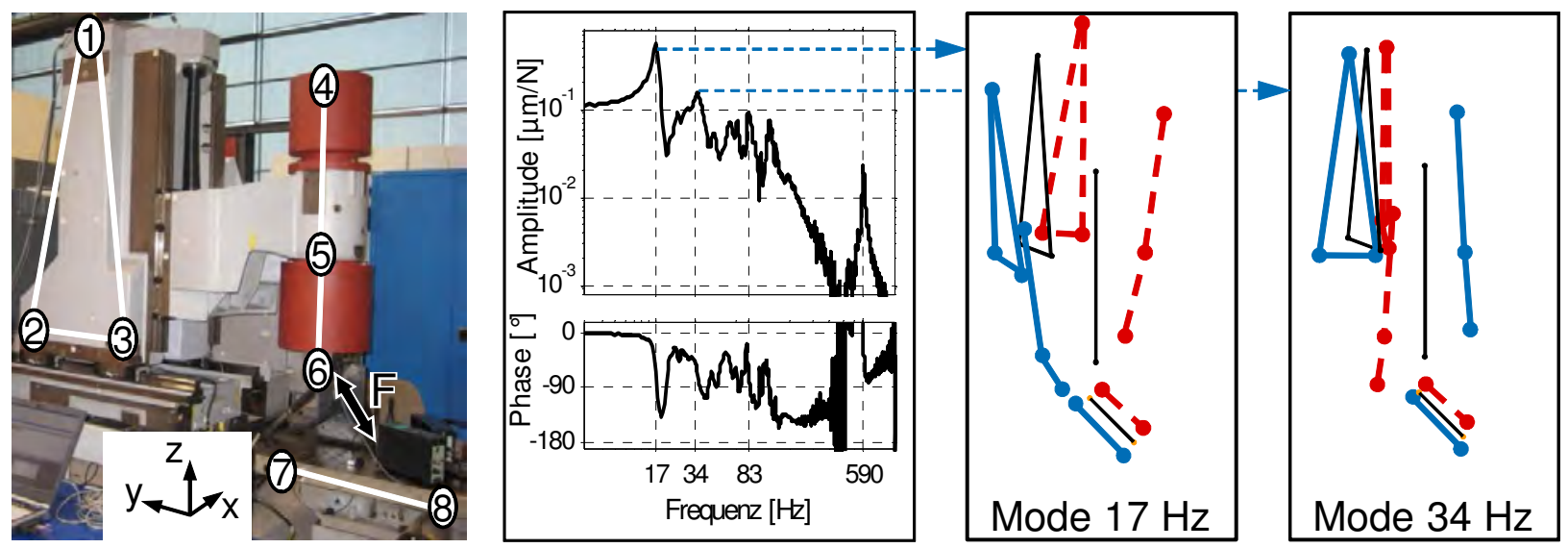

Bild 9 Stark reduzierte Modalanalyse mit einem Tracking-Interferometer

\section{Zusammenfassung}

Modalanalysen werden heute mit Hilfe von Beschleunigungssensoren und 3D-Laservibrometern durchgeführt, je nachdem ob es sich um offene Oberflächen oder um umhauste Strukturen handelt, bzw. eine erhöhte Genauigkeit verlangt ist. In dieser Arbeit wird eine neue Kombination an physikalischen Messprinzipien zur 3D-Schwingungsmessung, eine neue Vorgehensweise zur Erstellung der Modalanalyse mit teilautomatisierten und parallelisierten Modellerstellung und Schwingungsmessung sowie ein modifiziertes Tracking-Interferometer als neues Messgerät für die Erstellung von Modalanalysen vorgestellt.

Zunächst wird die Geometrie des Laserstrahls im optischen Reflektor dargestellt und gezeigt, dass Bewegungen des Reflektors in Strahlrichtung und quer zur Strahlrichtung unterschiedliche, deutlich separierte Einflüsse und die Drehung des Reflektors keinen Einfluss auf den Strahlverlauf haben. Für die Dynamikmessung muss der Reflektor also nicht aufwendig ausgerichtet werden. Vergleichsmessungen an einer Fahrständermaschine mit Laservibrometer, Weg- und Beschleunigungssensoren bescheinigen dem Tracking-Interferometer sehr gute Ergebnisse bis zu einer Frequenz von ca. $250 \mathrm{~Hz}$. Bei höheren Frequenzen sinkt die Verlagerungsamplitude und PSD-Messungen quer zur Strahlrichtung sind aufgrund von internen Mehrfachreflektionen des Laserstrahls stark verrauscht. Da dieses Rauschen jedoch stark mit einem Interferometersignal korreliert ist, wird in den folgenden Forschungsarbeiten eine rechnerische Kompensation dieses Rauschens angestrebt, andererseits werden Untersuchungen zur Unterdrückung dieser Mehrfachreflektionen durchgeführt.

Das Tracking-Interferometer wird ebenfalls zur Vermessung des geometrischen Modells der zu untersuchenden Struktur verwendet. Durch Referenzreflektoren können verschiedene Positionen und Ausrichtungen des Gerätes vermessen und zu einander ins Verhältnis gesetzt werden, so dass sowohl die gesamte Maschinenstruktur als auch die Schwingungsrichtungen anschlieBend in ein einheitliches Koordinatensystem transformiert werden. Die Koordinatentransformation der Schwingungsmessung wurde demonstriert. Zusätzlich wurden zwei Messverfahren zur Ermittlung der Absolutdistanz ohne Einsatz einer Absolutdistanzmesseinheit vorgestellt. Diese konnten bisher noch nicht die erforderliche Genauigkeit erreichen.

Eine erste stark reduzierte Modalanalyse einer Fahrständermaschine wurde vorgestellt.

Aktuelle Forschungs- und Entwicklungsanstrengungen beschäftigen sich mit der Verbesserung der PSD-Messungen und der Verfahren zur Messung der Absolutdistanz. Des Weiteren wird eine Anwendungssoftware zu Demonstrationszwecken entwickelt. Es ist das erklärte Ziel die Eignung und die Konkurrenzfähigkeit des Tracking-Interferometers als praktisches Werkzeug zur Erstellung von Modalanalysen nachzuweisen 


\section{Danksagung}

Die hier vorgestellten Arbeiten werden innerhalb des „DynaTrac“-Projektes durchgeführt. Das Projekt wird vom Bundesministerium für Bildung und Forschung (BMBF) im Rahmenkonzept „Forschung für die Produktion von Morgen“ gefördert und vom Projektträger Karlsruhe (PTKA) betreut.

\section{Literaturverzeichnis}

1. Ewins, D.J. (2000): Modal Testing, Theory, Practice and Application. Research Studies Press LTD, Baldock

2. Brecher, C.; Esser, M., Witt, S. (2009): Interaction of Manufacturing Process and Machine Tool, CIRP Annals - Manufacturing Technology, Jg., 58, Nr. 2, S. 588-612

3. Aurich, J.C.; Biermann, D.; Blum, H.; Brecher, C.; Carstensen, C.; Denkena, B.; Klocke, F.; Kröger, M.; Steinmann, P.; Weinert, K. (2009): Modelling and simulation of processmachine interaction in grinding. Prod. Eng. Res. Devel. Band 3, S. 111-120

4. Brecher, C.; Weck, M. (2006): Werkzeugmaschinen, Band 5. Messtechnische Untersuchung und Beurteilung, dynamische Stabilität. Springer, Berlin

5. Oliver, D.E. (2009): Tutorial: 3D Scanning Vibrometry for Structural Dynamics Measurements. Präsentation auf der IMAC-XXVII, 9.-12. Februar 2009, Orlando

http://www.sem.org/PDF/Oliver_3D\%20Scanning\%20Vibrometry.pdf . Abgerufen am 30.01.2012

6. Pingle, P.; Sailhamer, J.; Avitabile, P. (2009): Comparison of 3D Laser Vibrometer and Accelerometer Frequency Measurements, Proceedings of the IMAC-XXVII, 9.-12. Februar 2009, Orlando

7. Lau, K.; Hocken, R.J.; Haight, W.C. (1986): Automatic laser tracking interferometer system for robot metrology. Precision Engineering, Band 8/1, S. 3-8

8. Kniel, K. (2007): Ein Beitrag zur Entwicklung eines laserinterferometrischen Trackingsystems für die Genauigkeitssteigerung in der Koordinatenmesstechnik. Dissertation, TU Ilmenau

9. Schmitt, R.; Jatzkowski, P.; Schwenke, H.; Warmann, C. (2009): Advances in the error mapping of machine tools and coordinate measurement machines (CMMs) by sequential multilateration. Proceedings of the 9th LAMDAMAP, 30. Juni - 2. Juli 2009, Brunel University, West London, S. 452-461

10. Jatzkowski, P. (2011): Ressourceneffiziente Kalibrierung von 5-AchsWerkzeugmaschinen mit Tracking-Interferometern. Dissertation, RWTH Aachen University.

11. Brecher, C.; Guralnik, A.; Bäumler, S. (2012) Measurement of structure dynamics using a tracking-interferometer, Prod. Eng. Res. Devel., Band 6/1, S. 89-96

12. Brecher, C.; Bäumler, S.; Wissmann, M.; Guralnik, A. (2012): Modal Testing using Tracking-Interferometers, Proceedings of the SEM IMAC XXX Conference 30. Januar -2. Februar 2012, Jacksonville, Band 6

13. Brandt, A.(2011): Noise and Vibration Analysis. Wiley, Chichester 